\title{
STRUCTURAL DYNAMIC ANALYSIS OF BIOINSPIRED CARBON FIBRE/POLYETHYLENE MAV WINGS
}

\author{
David Kumar ${ }^{*}$, Mit Shah, P M Mohite, Sudhir Kamle \\ Department of Aerospace Engineering, \\ Indian Institute of Technology Kanpur, Uttar Pradesh, India-208016 \\ *davideiitk.ac.in
}

\begin{abstract}
Flapping wing micro air vehicles (FWMAVs) are small unmanned aircrafts or flying robots which are intended to be used for surveillance, reconnaissance, biochemical sensing, targeting, tracking, etc. To perform such missions, MAVs are required to do some specific operations such as hovering; slow and high speed flying; quick landing and take-off, etc. During flapping motion through surrounding air, wings experience inertial and aerodynamic forces. For making successful flights in such conditions, wings must have properties such as flexibility, strength, low weight, long fatigue life, etc. For producing such properties, wing material plays a crucial role. Most of the research related to MAVs is based on aerodynamics and controls. Present research is based on materials and structural aspects of flapping wings. Here materials used are carbon fibres for making wing skeleton and polyethylene for wing membrane. The design for the wing of $113.8 \mathrm{~mm}$ length is inspired from giant hummingbird's wing. The wing sketch was developed in gambit software by taking position data, generated using digitizer, from printed image of hummingbird wing. Developed sketch was printed and used, as a guide, for making the wing skeleton. The polyethylene film with adhesive was laminated on the skeleton at $150{ }^{\circ} \mathrm{C}$. Natural frequencies, nature of mode shapes, and damping characteristics of fabricated wings are determined here.
\end{abstract}

\section{KEYWORDS}

FWMAVs, Bioinspired Robotics, Hummingbirds, Structural Dynamics, Carbon Fibres, Polyethylene

\section{INTRODUCTION}

A significant progress has been noticed in various miniaturization fields. This technological advancement motivated DARPA, USA to initiate Micro Air Vehicle (MAV) development program. MAVs are characterized by the type of wings used, namely flapping, fixed, and rotary wing MAVs. Flapping wing MAVs can fly at low speeds or hover, and are agile and highly manoeuvrable. They generate lift and thrust using their wings only which is advantageous in terms of efficiency. MAVs are intended to be used for defence purposes including surveillance, targeting, tracking, remote sensing, reconnaissance operations, etc. Natural flyers, the original masters of flapping flight, are the best source of inspiration for making successful flapping wing MAVs or bioinspired flying robots. They are still more efficient than any man made flyer. Among the numerous examples of highly efficient flapping flyers that exist in nature, the hummingbird is chosen as the biological inspiration for the present study. The hummingbirds, because of their 
specific flapping mechanism and wings, have excellent characteristics such as long duration hovering, backward motion, high agility, etc. Hummingbirds can rotate their wings up to $180^{\circ}$ and create reverse camber during up stroke.

For increasing efficiency, many agile natural fliers do flapping motions through resonant excitation with the help of muscle tissue which excites the wings in vibratory mode [1-4]. Raney and Slominski [1] have studied the flight of natural fliers generating lift through "resonant excitation". Many researchers $[1,5,6]$, working on MAV flapping wings, used carbon fibres as wing frame with different polymeric material as wing membrane. Hummingbird wings have been taken as bio inspiration for making wings $[1,5]$. The analysis of flapping wing flight involves three components, kinematics, structural dynamics and aerodynamics [7]. Significant amount of research has been done on kinematics, controls, and aerodynamics fields related to MAVs. The structural dynamic analysis of MAV flapping wings is important for making a wing with desired structural characteristics. Chakravarty and Albertani [8] did experimental and finite element modal analysis of flexible latex membrane which was attached to an elliptical planform shape aluminium ring. To validate finite element model, they did experimental study in vacuum. Chakravarty [9] did another similar study, in air and in vacuum or reduced pressure environment, of hyperelastic radially pre-stretched membrane attached to rigid circular disk. It was found that the natural frequency increases with pre-stretched level and in vacuum. In vacuum, damping due to air is not present; therefore, natural frequency values and amplitude of vibration increase. Ha et al [10] developed and did modal analysis on artificial wing mimicking an allomyrina dichotoma beetle's hind wing. They found out the natural frequencies, nature of mode shapes, and damping ratios using fast Fourier transform analyser and laser displacement sensor. Sladek et al [11] did aerodynamic and structural dynamic analysis of wings, and used the results for determination of the repeatability of their wing manufacturing technique.

The objective of present study is to do structural dynamic analysis of carbon fiber/polyethylene hummingbird inspired MAV flapping wings. A hummingbird inspired wing has been designed and developed. Modal analysis has been done to obtain natural frequencies and mode shapes whereas damping analysis has been done to find the damping ratio of wings.

\section{DEVELOPMENT OF WINGS}

For making successful MAV, the wings have to be designed properly and appropriate materials have to be used for fabrication. The wings should be light in weight; structurally flexible and strong; able to flap at desired flapping frequencies and amplitude. Here the bioinspired wing designing and fabrication will be discussed.

\subsection{Wing Designing}

Natural flyers are the best source of inspiration for making successful MAVs. The hummingbirds have excellent characteristics such as long duration hovering, backward motion, high agility, etc. Hummingbirds can rotate their wings up to $180^{\circ}$ and create reverse camber during upstroke to generate lift. The wing designing was done using Gambit software. A printed image of giant hummingbird wing, see Fig. 1(a), was chosen for recording the dimensions for generating wing profile data. A digitizer was used to generate the data points from printed image. The computational wing profile sketch, shown in Fig. 1(b), was developed by importing data points into Gambit. 

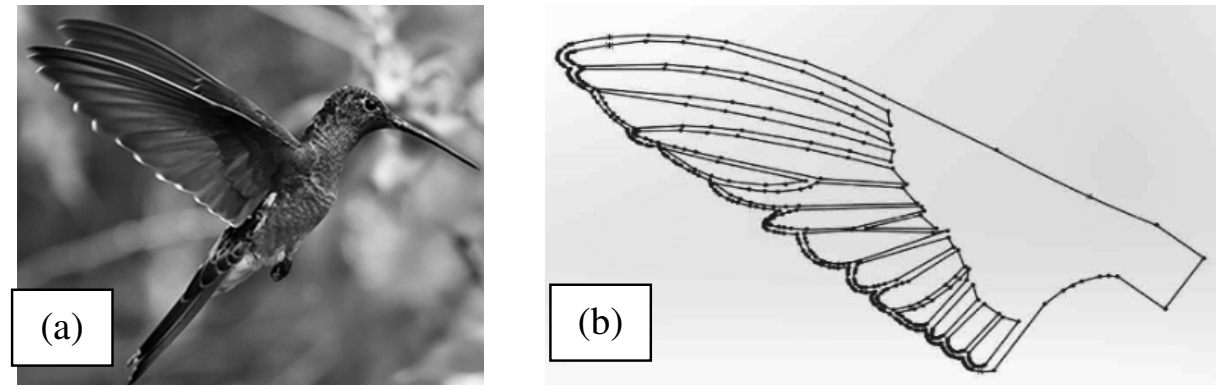

Figure 1. Designed wing 2D skeleton, (a) Giant hummingbird photo, (b) Developed sketch

The length and wing area of developed wing sketch are $113.8 \mathrm{~mm}$ and $3465.14 \mathrm{~mm}^{2}$ (excluding clamping part), respectively. The design parameters are in the hummingbirds range. The wing length of actual giant hummingbird ranges up to $135 \mathrm{~mm}$.

\subsection{Wing Manufacturing}

The manufacturing technique should be repeatable to produce wings with similar characteristics. The wings with different characteristics can create asymmetric flapping condition. The wing sketch, shown in Fig. 1(b), was used to make wing skeleton. The skeleton image was printed and used as a guide for placement of carbon fiber strands. The carbon fiber strands were first dipped into epoxy (DGEBA resin/TETA hardener in 10:1 ratio) solution before placing on the skeleton image. After placing strands the skeleton was allowed to cure for 24 hour at room temperature. For making complete wing a document lamination low density polyethylene (LDPE) film with adhesive was used. Using a lamination machine the developed skeleton was laminated at $150{ }^{\circ} \mathrm{C}$ (higher than melting temperature of LDPE) with the film. Developed wing is shown in Fig. 2. The weight of wing (average of three) is $0.97 \mathrm{gm}$.

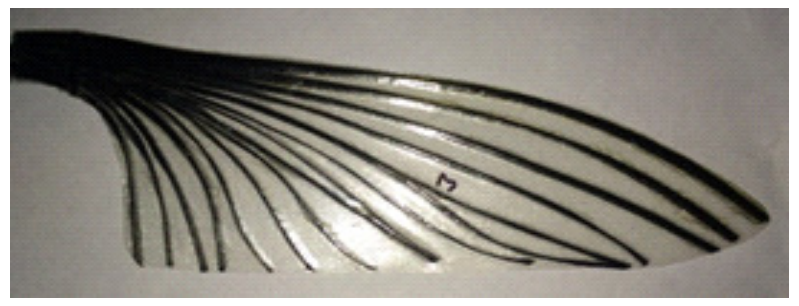

Figure 2. Final carbon fiber/polyethylene wing

\section{TESTING OF WINGS}

The developed wings are tested for their modal and damping characteristics. The aim of modal analysis is to find first three resonance modes and corresponding mode shapes whereas damping analysis is done to obtain the damping ratio of wings.

Many agile natural flyers do flapping motions through resonant excitation with the help of muscle tissue which excites the wing in vibratory mode [1-4]. At resonance the amplitude increases so does the lift and thrust. This paper is mainly focused on finding the fundamental or first modal frequency and shape because the amplitude for this mode is always higher than other modes. The power needed to vibrate/flap the wing in the first resonance mode will be lesser than other modes. Therefore flapping at first mode will increase the efficiency of MAVs as compared to flapping at other modes. 


\subsection{Modal Analysis}

The modal analysis is done with the help of electromagnetic shaker with power amplifier, laser displacement shaker, stroboscope, and high speed camera (see Fig. 3). Electro-magnetic shaker excites the wing at different frequency and amplitude which are controlled by power amplifier. Laser displacement sensor is used to observe the live displacement of vibrating wing to find the resonance mode. NI-DAQ cards with LabVIEW program are used to receive the live displacement data from laser sensor and show on the computer screen. At resonance the displacement of wing, observed on computer screen, becomes high as compared to lower and higher frequency excitations. Stroboscope is used to identify the nature of mode shapes. The high speed camera (Olympus i-Speed TR of $10000 \mathrm{fps}$ capability) is used to record the mode shapes of wing at resonance conditions.

\subsection{Damping Analysis}

Damping study of the flapping wings is an important aspect of study because the wing must be able to reduce unwanted vibrations due to sudden wind gusts without losing control. This requires an estimate of the logarithmic decrement $(\delta)$ and the damping ratio $(\xi)$ of the wing. The mathematical relations used for calculating these quantities are:

$$
\xi=\frac{1}{\delta=\ln \left(\frac{x_{1}}{x_{2}}\right)} \frac{1}{\sqrt{1+\left(\frac{2 \pi}{\delta}\right)^{2}}}
$$

Where, $\mathrm{x}_{1}$ and $\mathrm{x}_{2}$ are the first and second maximum positive amplitudes of time wing-tip displacement history plot of wing after giving an initial displacement. An experimental setup using laser displacement sensor, LabVIEW program for data acquisition and a wing clamp was made for this analysis. The wing was clamped at the root section and excited by a sudden jerk at the tip. Tip displacements were measured via laser sensor and recorded as a function of time to study the damping characteristics.

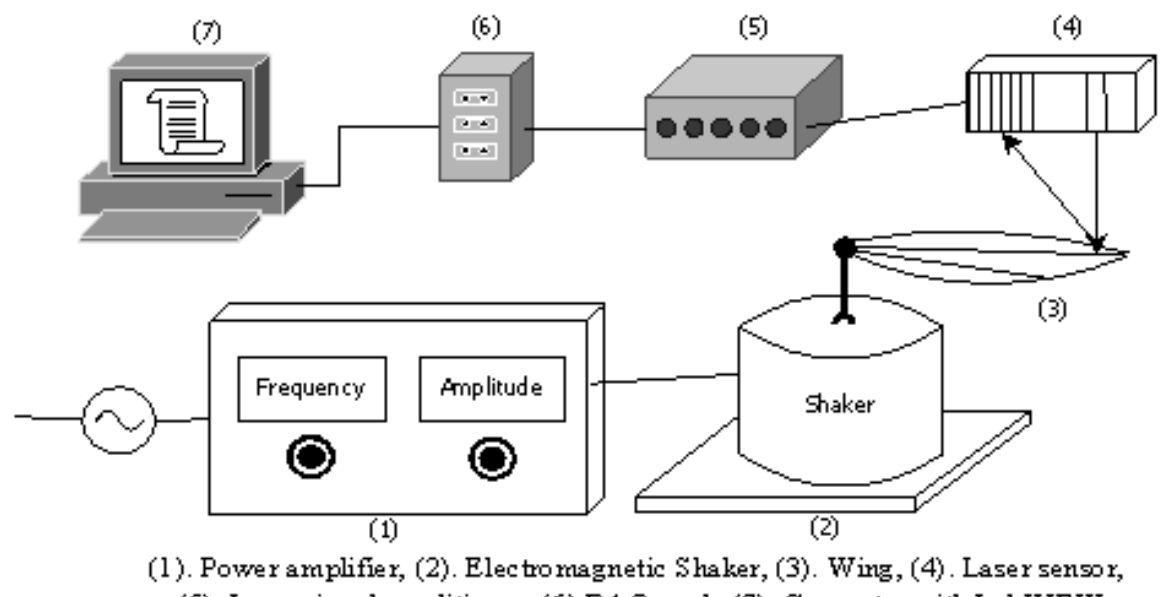

(5). Laser signal conditioner, (6) DAQ cards (7). Computer with LabVIEW

Figure 3. Experimental setup 


\section{RESULTS AND DISCUSSION}

\subsection{Modal Analysis Results}

The natural frequencies and mode shapes results of the developed wings are discussed here.

\subsubsection{Natural Frequency}

The natural frequencies for all three wings are shown in the Table 1. Natural frequencies of three similar wings are compared for repeatability determination of used wing manufacturing technique. The small values of standard deviation (SD) show that the wing manufacturing is repeatable to produce the wings with similar structural dynamics. The fundamental mode of all wings is at $26 \mathrm{~Hz}$ which means the wing manufacturing technique is $100 \%$ repeatable for this mode.

To increase the efficiency of bioinspired structure the wings should be flapped close to the first fundamental frequency. The hummingbird inspired resonance flapping mechanism should be used to flap theses wings to get the benefit of high amplitude at resonance. This resonance frequency is in the flapping/beat rate range of actual hummingbirds. The beat rate range of hummingbirds is 12-100 Hz [12].

Table 1. Natural frequency results.

\begin{tabular}{|c|c|c|c|c|c|}
\hline Mode & Wing-1 & Wing-2 & Wing-3 & SD & Mean \\
\hline Mode 1 & 26 & 26 & 26 & 0.00 & 26.00 \\
\hline Mode 2 & 90 & 84 & 91 & 3.78 & 88.33 \\
\hline Mode 3 & 144 & 132 & 131 & 7.23 & 135.67 \\
\hline
\end{tabular}

\subsubsection{Mode Shapes}

The mode shapes, for all first three resonance modes, have been captured through high speed camera at $2000 \mathrm{fps}$. The amplitude of flapping is directly connected to efficiency of system. Higher amplitude increases the efficiency. The fundamental mode has highest amplitude as compared to others modes (see Fig. 4, Fig. 5 and Fig. 6). The wing was excited at same base amplitude for all modes to compare the displacement gained by wing at resonance frequencies. Another important point to note here is that the wing is able to vibrate/flap at high amplitude, see Fig. 4. The wing-tip displacement is very high as compared to base amplitude applied at clamped wing-root.

The wing was continuously vibrated for $30 \mathrm{~min}$ at wing-tip amplitude $\sim 72 \mathrm{~mm}$ at first mode to observe its fatigue characteristics. The wing performed well without any failure. No cracks/damages were observed. A more detailed experimental study has to be done for fatigue characterization. 
International Journal of Recent advances in Mechanical Engineering (IJMECH) Vol.3, No.4, November 2014
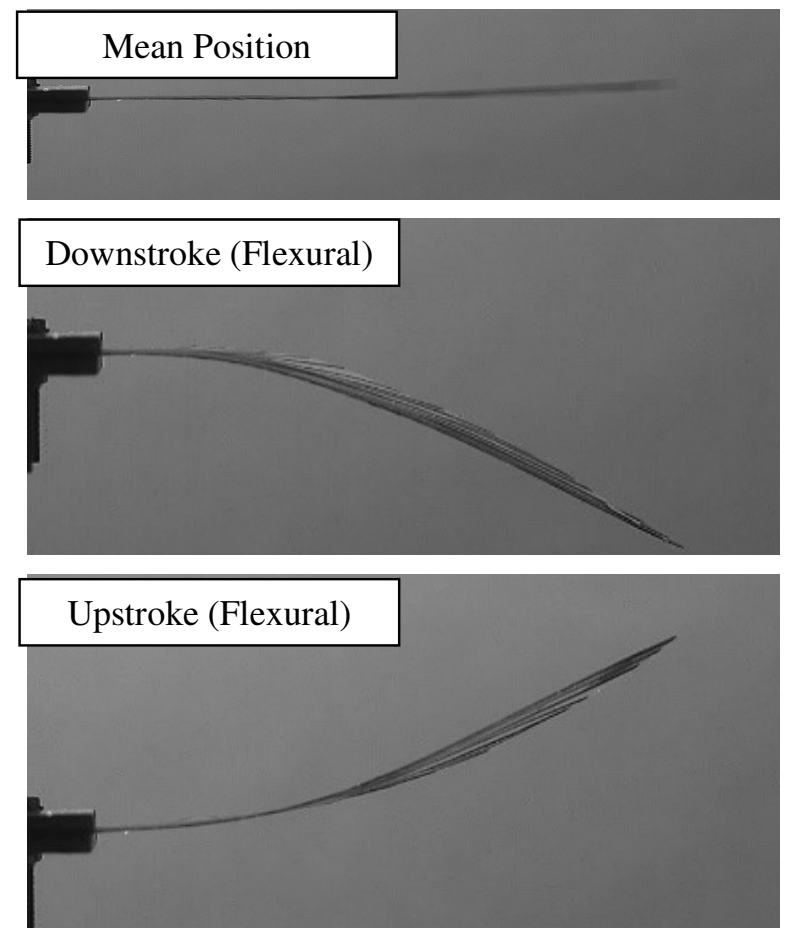

Figure 4. Mode shape of fundamental mode: Bending mode

Downstroke (Twisting)
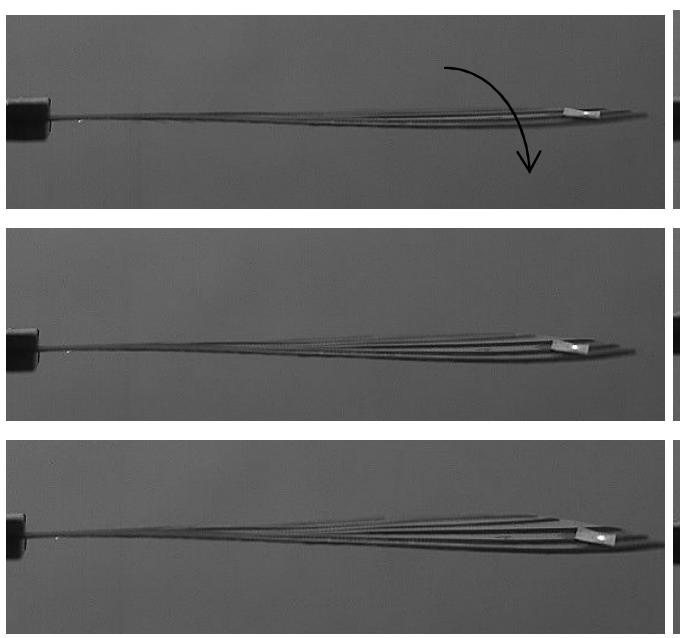

Upstroke (Twisting)
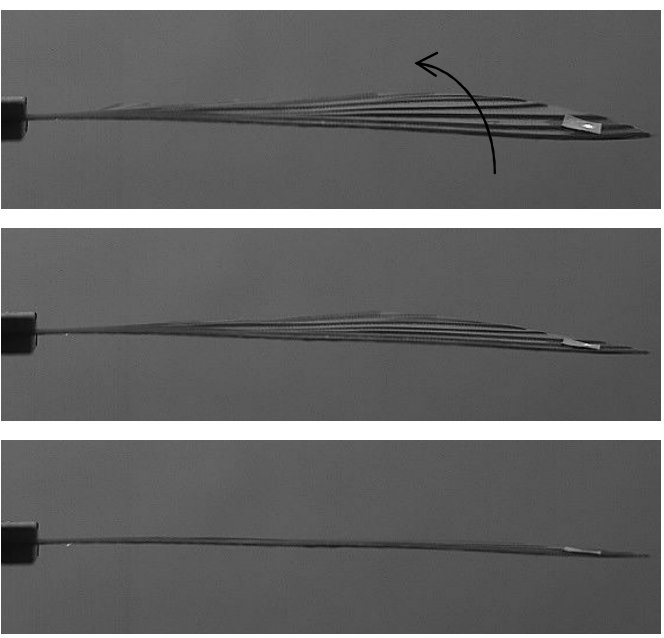

Figure 5. Mode shape of second mode: Twisting mode 
Downstroke (Flexural)
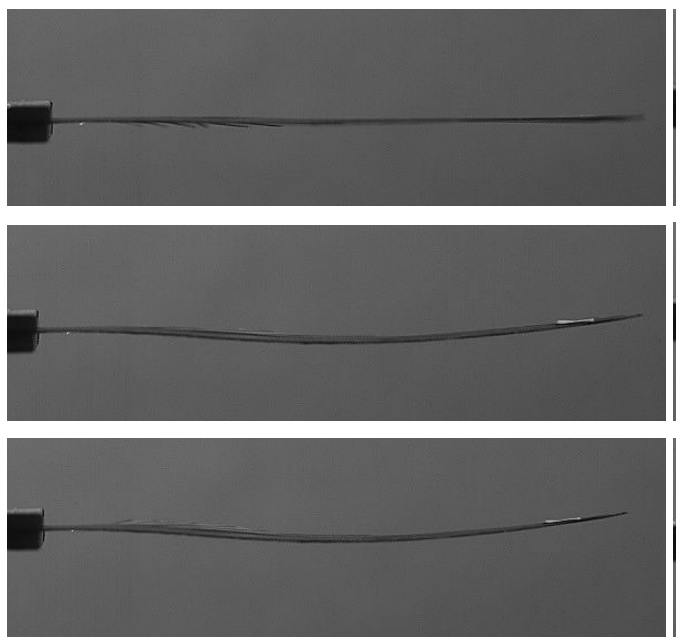

Upstroke (Twisting)
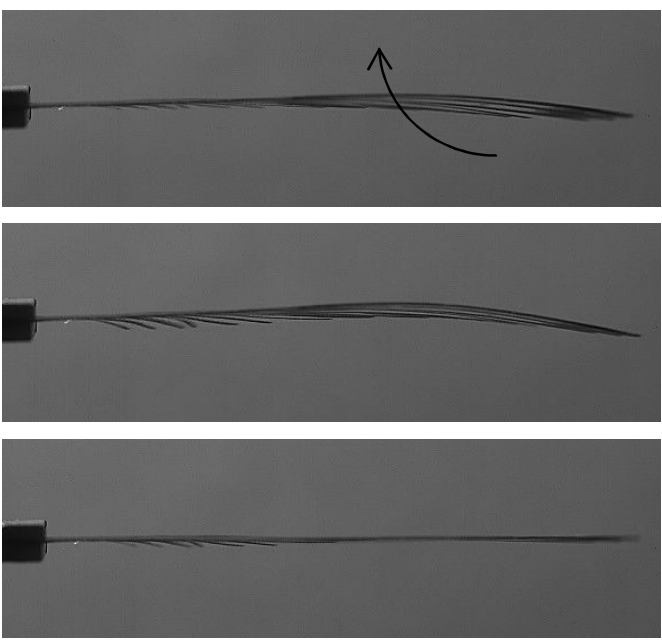

Figure 6. Mode shape of third mode: Coupled mode

\subsection{Damping Characteristics}

The Table 2 shows the damping characteristics of the wings. The average damping ratio for the three wings is 0.0702 , which means that the wings structure has under-damped nature.

Table 2. Damping analysis results.

\begin{tabular}{|c|c|c|c|c|c|}
\hline Property & Wing-1 & Wing-2 & Wing-3 & SD & Mean \\
\hline $\boldsymbol{\delta}$ & 0.3579 & 0.3977 & 0.5712 & 0.1134 & 0.4422 \\
\hline $\boldsymbol{\xi}$ & 0.0568 & 0.0632 & 0.0905 & 0.01789 & 0.0702 \\
\hline
\end{tabular}

The standard deviation here is large as compared to average value. This is because of less order of magnitude of damping results. Maintaining repeatability is tough in this case.

The wing is subjected to two types of damping, namely the structural damping due to the system itself and due to the surroundings. The system damping is due to the wing structure itself or viscoelastic nature of wing material and the surrounding damping is due to fluid or air interactions. The damping due to wing structure has been calculated here. The main supporting component, the skeleton, of wings is made of carbon fibres which are known for their high elastic modulus and strength. The damping ratio is very small which indicates that the wing structure has more elastic nature than viscous.

\section{CONCLUSION}

The objective of this study was the development and structural dynamic analysis of hummingbird inspired carbon fiber/polyethylene wings. The wing is successfully designed and developed. Three wings were developed for determination of repeatability of wing manufacturing technique. Modal and damping analysis have been done of developed wings. The wings have fundamental mode at $26 \mathrm{~Hz}$, in the flapping rate range of actual hummingbirds, with $100 \%$ repeatability. The amplitude of fundamental mode is higher than other mode. The damping ratio of wings was determined to be 0.07 which indicates that the wings are under-damped structures. 
For improving the efficiency of a bioinspired structure, the flapping should be done close to the resonant frequency. Therefore the developed wings should be flapped using hummingbird inspired resonance flapping mechanism at the fundamental mode obtained here.

The developed wings are light and structurally strong, and can be vibrated/flapped at very high amplitude and desired frequency for a very long time. A fatigue characterization study has to be done to confirm these characteristics of wings. In the present study the complex shape hummingbird inspired wings are developed successfully. Similarly, any complex shape small size structures such as other birds or insect inspired wings, MAVs parts etc. can be developed. This study is beneficial for aerodynamicists. The study attempts to produce the wings with good structural properties.

\section{ACKNOWLEDGEMENTS}

The authors would like to thank NP-MICAV (Sanction Ref No: DARO/081102072/M/I/SM-02) for partial financial support to carry out this work. The authors also thank Mr. Lavendra Singh from Structures Lab, Department of Aerospace Engineering for his technical assistance.

\section{REFERENCES}

[1] D.L. Raney and E.C. Slominski, (2004) "Mechanization and control concepts for biologically inspired micro air vehicles", Journal of Aircraft, Vol. 41, No. 6, pp1257-1265.

[2] R. Dudley, (2002) "The biomechanics of insect flight: form, function, evolution", Princeton University Press.

[3] M. Dickinsonl, (2001) "Solving the mystery of insect flight", Scientific American, Vol. 284, No. 6, pp48.

[4] M. J. Patil, (2003) "From fluttering wings to flapping flight: The energy connection", Journal of Aircraft, Vol. 40, No. 2, pp270-276.

[5] P. Zdunich, D. Bilyk, M. MacMaster, D. Loewen, J. DeLaurier, R. Kornbluh, T. Low, S. Stanford, and D. Holeman, (2007) "Development and testing of the mentor flapping-wing micro air vehicle", Journal of Aircraft, Vol. 44, No. 5, pp1701-1711.

[6] R.J. Wood, "Design, fabrication, and analysis of a 3dof, $3 \mathrm{~cm}$ flapping-wing MAV", In Intelligent Robots and Systems, 2007. IROS 2007. IEEE/RSJ International Conference on, 2007.

[7] P. Wu, B.K. Stanford, E. Sllstrm, L. Ukeiley, and P.G. Ifju, (2011) "Structural dynamics and aerodynamics measurements of biologically inspired flexible flapping wings", Bioinspiration and Biomimetics, Vol. 6, No. 1, pp016009.

[8] U.K. Chakravarty and R. Albertani, (2011) "Modal analysis of a flexible membrane wing of micro air vehicles", Journal of Aircraft, Vol. 48, No. 6, pp1960-1967.

[9] U.K. Chakravarty, (2013) "Analytical and finite element modal analysis of a hyperelastic membrane for micro air vehicle wings", Journal of Vibration and Acoustics, Vol. 135, No. 5, pp051004.

[10] N.S. Ha, T. Jin, and N. S. Goo, (2013) "Modal analysis of an artificial wing mimicking an allomyrina dichotoma beetle's hind wing for flapping-wing micro air vehicles by noncontact measurement techniques", Optics and Lasers in Engineering, Vol. 51, No. 5, pp560 - 570.

[11] N.J. Sladek, M.L. Anderson, and R.G. Cobb, "Aero and structural-dynamic repeatability of a novel MAV wing manufacturing process", In AIAA Atmospheric Flight Mechanics Conference, 2011.

[12] Information on "http://en.wikipedia.org/wiki/Hummingbird". 


\section{AUTHORS}

Dr Sudhir Kamle is Professor at Department of Aerospace Engineering, Indian Institute of Technology Kanpur. Research Interests: Experimental Stress Analysis, Smart Materials, Structural Dynamics Email: ka mle@iitk.ac.in

Dr P M Mohite is Assistant Professor at Department of Aerospace Engineering, Indian Institute of Technology Kanpur. Research Interests: Damage Mechanics of Laminated Composites, Composites, Finite Element Analysis

Email: mohite@iitk.ac.i n

David Kumar is graduate student at Departmen t of Aerospace Engineering, Indian Institute of Technology Kanpur. Research Interests: Structural Dynamics, Nanocomposites, Dynamic Material Characterization, MAVs Email:david@iitk.ac.in

Mit Shah is under graduate student at Department of Aerospace Engineering, Indian Institute of Technology Kanpur. Research Interests: Structural Dynamics, A eromodelling, LabVIEW Programming, MAVs Email: mmshah@iitk.ac.in
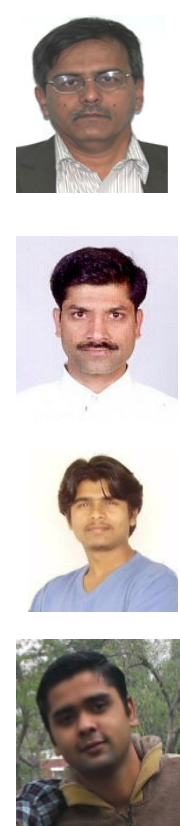